\title{
REFLEXIONES SOBRE CATÁLOGOS Y CATALOGACIÓN DE MANUSCRITOS ÁRABES
}

\author{
Nuria TORRES SANTO DOMINGO \\ Biblioteca Islámica "Félix María Pareja», AECI
}

En octubre de 1987, poco tiempo después de empezar a trabajar en la Biblioteca Nacional, tuve la oportunidad de acercarme, por primera vez, a los problemas que surgen a la hora de la catalogación de manuscritos. Desde entonces han sido varios los períodos en los que he trabajado con este tipo de materiales y he conocido los problemas relativos no sólo a su tratamiento para una adecuada conservación, preservación, restauración y microfilmación, sino también al tratamiento documental, catalogación y descripción bibliográfica. Se me ha encargado que me ocupe de este último aspecto, no tan brillante como el anterior, pero sin el cual sería imposible la realización de catálogos y, aunque no me considero en absoluto una experta en el tema, ofrezco, al menos, unas reflexiones producto de mi modesta experiencia.

Partiendo desde el principio, no está de más recordar que lo que se conoce por manuscrito es un texto escrito a mano ${ }^{1}$, sin intervención de la imprenta o cualquier otro modo de reproducción. Según quien escribe el texto, el manuscrito adopta una característica u otra que influye en sú denominación. Así, se denomina manuscrito autógrafo si la letra es la del propio autor, ya esté firmado o no; apógrafo si, por el contrario, es un texto copiado del original del autor. Según su estado físico, podemos hablar de manuscrito acéfalo, al que le falta el principio; mutilado, si le han desaparecido cuadernos o bifolios. Según el estado literario, podemos hablar de manuscrito original, copia, borrador, etc.

De la misma manera hablamos de códices, aunque es un término un poco impreciso, cuando nos referimoś a los manuscritos anteriores a la aparición de la imprenta, formado con hojas reunidas en cuadernos cosidos a lo largo del pliegue. La importancia de este tipo de manuscritos es tal que en torno a ellos, y a partir sobre todo de mediados de este siglo, se ha desarrollado la ciencia de la codicología que, junto con la paleografía, estudia el entorno completo que conlleva su realización, esto es, el soporte del texto, la tinta utilizada, el tamaño del manuscrito, la composición de los cuadernos, la caja de escritura, el tipo de escritura, etc.

\footnotetext{
${ }^{1}$ Sánchez Mariana, M., Introducción al libro manuscrito, Madrid, 1995, [p. 7]. También se puede aplicar a textos mecanografiados, según el punto 3.0 de las Reglas de catalogación, Madrid, 1995, p. 109.
} 
La aparición de la imprenta no produjo, sin embargo, la desaparición del libro manuscrito, especialmente los que vamos a analizar, y es bastante frecuente encontrar obras manuscritas hasta bien entrado el siglo XIX.

Por último, hay que aclarar que el manuscrito misceláneo, de presencia asidua en todas las colecciones españolas, sobre las que nos hemos basado para realizar estas notas, es aquél que contiene en un mismo volumen encuadernado varias piezas de diversa procedencia, es decir, tiene una unidad física pero no de contenido. Se diferencia de una colección de manuscritos en que esta última contiene diversos manuscritos con una unidad diferenciadora y cuyo elemento unificador es su procedencia; por ejemplo, un epistolario o incluso documentos propios de archivos.

El segundo elemento que caracteriza nuestro estudio se refiere a la lengua árabe, una lengua semítica escrita de derecha a izquierda en caracteres no latinos. Además de ser la lengua litúrgica de los musulmanes, es la primera en la que se encuentra escrito el desarrollo del pensamiento de la civilización islámica en todas sus vertientes: religiosa, científica, filosófica, histórica y, por supuesto, literaria. Con caracteres propios del árabe se escribe también la lengua persa y, hasta 1928, la lengua turca.

La unión de los elementos anteriores nos lleva a introducir el tema que nos va a ocupar en estas líneas y que se refiere a los manuscritos árabes. Antes de nada me gustaría hacer la reflexión de que hablar de manuscritos en árabe o manuscritos escritos en árabe no significa necesariamente hablar de manuscritos de tema islámico. Aunque es bien cierto que la mayoría de los textos escritos en caracteres árabes - bien en lengua árabe, persa o turca - contienen una temática islámica, también lo es que se encuentran textos en la misma escritura de temática distinta, evangelios, diccionarios, etc., que no se pueden encuadrar de ninguna manera bajo la misma denominación ${ }^{2}$. Pensemos de la misma manera en la literatura aljamiada, lengua española escrita en caracteres árabes, reflejo de una dramática situación vivida en España por los herederos de una cultura y una lengua que estaban condenados a perder.

Por mi propia especialización como profesional no sólo de la información, sino también del libro y bibliotecas, he podido seguir de cerca un debate que se plantea desde hace algún tiempo respecto a cuál debe ser la actuación más adecuada sobre los manuscritos árabes en todo lo que se refiere al tratamiento documental. Casi toda la bibliografía disponible nos viene de manos de investigadores altamente especializados en la cultura árabe e islámica, y muy poca por los propios profesionales del sector del libro, hecho que no nos causa demasiada sorpresa, ya que el estudio de un manuscrito conlleva forzosamente

${ }^{2}$ A diferencia del término inglés Islamic manuscripts. Cf. World survey of Islamic manuscripts, ed. G. Ropper, London, 1992-1994, vol. 1, p. XV. 
múltiples incógnitas derivadas del mismo, como son: autoría, historia de textos y evidentes cuestiones lingüísticas y paleográficas, a los que hay que añadir un conocimiento atinado de las obras de referencia adecuadas.

Últimamente hay un gran interés por la salvaguarda de los manuscritos y su estudio en todos los aspectos posibles. Se trata de recuperar una herencia cultural que se encuentra repartida, en mayor o menor medida, en colecciones diversas de todas las bibliotecas del mundo.

Además de publicaciones periódicas muy especializadas ${ }^{3}$ hay un gran interés por la publicación de catálogos e inventarios de aquellas bibliotecas desconocidas y con menos posibilidades para dar a conocer sus fondos. Labor encomiable es la que lleva a cabo la fundación Al-Furqān Islamic Heritage Foundation establecida en Londres, dedicada única y exclusivamente a los manuscritos islámicos. Además de la edición de varios catálogos en los últimos tres años ${ }^{4}$, otra de sus actividades prioritarias ha estado enfocada en la reunión de expertos procedentes de todas las partes del mundo, para su formación en la noble y difícil tarea de catalogación de manuscritos, en una de cuyas sesiones tuve el privilegio de participar.

\section{MANUSCRITOS ÁRABES EN ESPAÑA}

En este estudio me voy a centrar fundamentalmente en la situación de las colecciones de manuscritos árabes que se conservan en este momento en España, así como de sus catálogos. Para ello me voy a basar en un estudio, editado precisamente por Al-Furqān, en una publicación que pretende dar información sobre colecciones de manuscritos en todo el mundo y que apareció entre 1992 y $1994^{5}$. Los datos referidos a España se cerraron en 1989, por lo que no se pudo incluir algún trabajo a punto de publicarse, al que hay que añadir otros posteriores, muy recientes.

\subsection{Análisis cuantitativo de las colecciones}

- Hay fundamentalmente dos grandes colecciones, alojadas la primera en la Biblioteca del Real Monasterio de El Escorial y la segunda en la Biblioteca Nacional de Madrid.

\footnotetext{
${ }^{3}$ Especializadas en el mundo árabe e islámico encontramos, por ejemplo, Manuscripta Orientalia, ISSN 1238-5018 (1995-); Manuscripts of the Middle East (1986- ); Nouvelles des manuscrits du Moyen Orient, ISSN 1167-2250 (1992- ), que incluye el magnífico Fichier des manuscrits moyenorientaux datés; a los que se puede añadir el título Gazette du livre médiéval, ISSN 0753-5015.

${ }^{4}$ Colecciones de Sofía, Bulgaria y Nigeria en 1995; Tombuctú entre 1995 y 1996.

${ }^{5}$ Torres Santo Domingo, N., "Spain", World survey of Islamic manuscripts, vol. 3, [95]-126.
} 
- Hay otras colecciones de tamaño medio en la Real Academia de la Historia de Madrid (371 mss.); en el Instituto de Filología, Departamento de Estudios Árabes del CSIC de Madrid (99 mss.); y en la Biblioteca del Monastir de Montserrat (ca. 100 mss.).

- Siguen a ésta varias colecciones pequeñas, algunas de ellas privadas, repartidas por toda la geografía española: Biblioteca Pública de Córdoba (61 mss.); Real Academia de Ciencias, Bellas Letras y Nobles Artes de Córdoba $(21 \mathrm{mss}$.); Biblioteca March de Mallorca (35 mss.); Fundación Lázaro Galdiano de Madrid (17 mss.); Biblioteca del Palacio Real de Madrid (12 mss.); Escuela de Estudios Árabes de Granada (40 mss.); Abadía del Sacromonte de Granada (21 mss.); Universidad de Sevilla (15 mss.); Archivo y Biblioteca Capitular de Toledo (26 mss.).

- Por último, se encuentran manuscritos aislados en colecciones muy concretas: Colección privada de Ocaña (6 mss.); $1 \mathrm{~ms}$. en la Universidad de Oviedo; 1 mss. conocido en la Biblioteca de la Universidad Complutense, Facultad de Filosofía y Letras, y al menos otros 2 mss. sin catalogar recientemente localizados en la misma BUC; 8 mss. en la Biblioteca Pública de Toledo; 7 mss. en la Biblioteca-Museo Balaguer de Vilanova i la Geltrù; 4 mss. en el Ayuntamiento de Villarrubia de Santiago; 1 ms. sin catalogar en la Academia de Jurisprudencia de Madrid; varios manuscritos en la colección particular de D. Miguel Asín, donada por sus herederos a la UNED.

En la Biblioteca Islámica "Félix María Pareja" de Madrid se han encontrado hasta el momento 3 manuscritos - uno de ellos es un diccionario árabelatín-castellano- y se han comprado otros dos. De éstos, un texto es de un autor andalusí y se trata de una obra de derecho malikí en copia realizada en Fez en el siglo XVII. También se han donado varios ejemplares, muy incompletos, procedentes de Tánger. Todos están sin estudiar.

Realmente, la situación que contemplamos no refleja en absoluto las circunstancias culturales e históricas de España, que convivió durante mucho tiempo con culturas distintas, entre ellas la árabo-islámica, cuya lengua vernácula era el árabe, sobradamente conocido y usado por todos los miembros de la población. Desgraciadamente, muchos manuscritos se perdieron, por causas y vicisitudes diferentes, y otros muchos de factura andalusí no se encuentran en España. Recordemos, a este respecto, las palabras de Antonio Almagro Cárdenas:

... aún faltan egemplares de valiosas obras debidas à la pluma de los moros españoles quienes las llevaron al ser expulsadas de España, y hoy se encuentran 
en las bibliotecas públicas y privadas de varias ciudades berberiscas, principalmente en Fez, Tetuán, Argel y Túnez ${ }^{6}$.

\subsection{Análisis cualitativo de las colecciones}

Es difícil ofrecer una valoración en este aspecto. De manera general, se estima que los manuscritos más interesantes son aquéllos en los que destacan una o varias cualidades estudiadas por la codicología, como es el soporte del texto, especialmente si se trata de pergamino, lo que atestiguaría la antigüedad del ejemplar, la iluminación, textos autógrafos, copias únicas aunque tardías u obras originales de autores andalusíes, etc.

En este sentido, sin duda alguna, la Biblioteca de El Escorial aloja la colección más sobresaliente, objeto de numerosos estudios, a los que hay que añadir algún texto interesante de la colección de la Biblioteca Nacional.

Hay pocos manuscritos andalusíes que se encuentren en España. Sin embargo, a través de otros catálogos sabemos de algunos de época andalusí, que se encuentran repartidos en otras colecciones extranjeras, europeas y orientales.

En España cobran especialísima importancia los manuscritos aljamiados, característica muy peculiar de la cultura española, de los que hay buenos ejemplares tanto en la Real Academia de la Historia como en el Instituto de Filología del CSIC de Madrid, sin olvidar tampoco los de la Biblioteca Nacional. A ellos hay que sumar colecciones pequeñas que aparecen un buen día entre muros, olvidados por el tiempo. La mayoría son copias de obras jurídicas y religiosas.

En general, hay una procedencia casi unidireccional de manuscritos: magrebíes; los orientales son más escasos en comparación con ellos.

Muchos de los manuscritos son meros fragmentos de textos, acéfalos y muy incompletos, lo que dificulta de manera extraordinaria su identificación.

Hay que señalar la presencia de pocos textos en persa - Fundación Lázaro Galdiano- y de turco - Biblioteca Nacional. Sin embargo, los estudios de lenguas semíticas en España no han seguido la tendencia mantenida en el resto de los países europeos, donde los estudios llamados orientales se completan con otras lenguas en las que hay manifestación islámica y donde hay especialistas, por tanto, en lenguas persas y turcas.

${ }^{6}$ Almagro Cárdenas, A., "Catálogo de los manuscritos árabes que se conservan en la Universidad de Granada", Actes du Onzième Congrès international des orientalistes, Paris, 1897, París, 3ème section, p. 46. 
Es curiosa la peripecia de muchos manuscritos de colecciones privadas, que han ido pasando de mano en mano a lo largo del tiempo y que, actualmente y por razones varias, se encuentran formando parte de una determinada colección, casi siempre pública, con capacidad suficiente para su alojamiento y tratamiento adecuado. Por ello existen muy pocas de carácter privado, entre ellas, la Biblioteca March y la de la Fundación Lázaro Galdiano, realmente excepcionales.

\section{ANÁLISIS DE LOS CATÁLOGOS EXISTENTES}

Tras un profundo estudio de los catálogos reseñados en el mencionado estudio ${ }^{7}$, la situación de los catálogos, en el sentido de construcción científica, es, a mi modo de ver, la siguiente:

- La mayoría de los considerados catálogos no lo son tal, ya que muchos de ellos son sólo inventarios, es decir, registros meramente indicativos con mención únicamente de autor y título que, en ocasiones, aparecen traducidos al español. Esto es bien cierto, sobre todo, con las primeras apariciones del siglo XVI. Su verdadera intención era dar a conocer la entrada de manuscritos en determinada colección; por lo tanto, no primaban los aspectos descriptivos, tan sólo los informativos.

- En cuanto a la disponibilidad y facilidad de manejo, para las grandes colecciones (El Escorial y Biblioteca Nacional) hay catálogos publicados separadamente en forma de volúmenes. Para el resto de las colecciones, mucho más pequeñas, los catálogos/inventarios se encuentran dispersos en distintas publicaciones científicas, lo que hace más difícil su localización y posterior consulta o estudio.

- Respecto a la transliteración utilizada, varía desde el principio (1760) hasta que se normaliza con el uso de la adoptada por las Escuelas de Estudios Árabes.

- En general, hay una falta completa de índices, casi siempre para El Escorial y Biblioteca Nacional.

- Se observa una falta de criterio único al describir colecciones. Esto se evidencia, por ejempo, en la indicación de los autores, que unas veces se hace por la šuhra y otras por el ism.

\footnotetext{
${ }^{7}$ Véase supra, nota 5.
} 
- Se aprecia también una ausencia de datos codicológicos que hagan posible el conocimiento de la factura del manuscrito en su posición absoluta y en su posición relativa al encuadrarse dentro de un posible origen común o factura.

- Muchas de las colecciones han sido objeto de varios estudios, listas, inventarios, catálogos a lo largo de la historia, y en el siglo XX se ha dado un repaso general a todas las colecciones.

- Respecto a los autores, la mayoría son arabistas españoles. Algunos se han dedicado con más profundidad que otros al estudio de manuscritos y encontramos sus nombres repetidos: Asín Palacios, Codera o el recientemente desaparecido Braulio Justel, que ejerció como Director de la Biblioteca de El Escorial.

De manera general, se puede afirmar que la realización de catálogos de colecciones de manuscritos escritos en árabe en España no se puede separar de la realización de catálogos de manuscritos de otras lenguas, latín o griego, por ejemplo. Como bien señala Manuel Sánchez Mariana en el prólogo a la guía de catálogos impresos de manuscritos de España ${ }^{8}$, la realización de catálogos de manuscritos empieza a plantearse en España, como en el resto de Europa, en el siglo XVI, y concretamente en torno a la biblioteca regia de El Escorial. Sin embargo, en estos primeros catálogos — como los de Benito Arias Montano en 1577 o el P. Sigüenza - y pese a una cierta teorización por parte de este último, no se encuentra todavía ningún intento de sistematizar los procedimientos de descripción bibliográfica.

Las primeras noticias de manuscritos son meras listas que dan noticia de la procedencia para su incoporación a la Biblioteca de El Escorial. Éstos han sido objeto de trabajo por Gregorio de Andrés en algunos artículos". Sirven, sobre todo, para conocer qué manuscritos se incorporaron a la mencionada biblioteca, y cuál era su procedencia. Se trataba fundamentalmente de alguna colección privada que se sumaba a la colección real que se estaba formando y que incluía distintos tipos de material manuscrito en diferentes lenguas, no solamente el árabe. Aunque en su momento tuvieron su importancia, que nunca se debe negar, desde la perspectiva de hoy no pueden considerarse material catalográfico, sino que deben considerarse como meramente informativas.

Tenemos que esperar al siglo XVIII y en torno a la Biblioteca Real, en época de Carlos III, cuando se manifiesta con claridad el reconocimiento del valor de los

\footnotetext{
${ }^{8}$ Martín Abad, J., Manuscritos de España: guía de catálogos impresos, Madrid, 1989, 8.

" Andrés, G. de, "El primer catálogo de manuscritos de la Biblioteca de El Escorial, 1572», Homenaje a Federico Navarro: miscelánea de estudios dedicados a su memoria, Madrid, 1973, 15-24; Torres Santo Domingo, Nuria, op. cit., 102-103.
} 
aspectos codicológicos de los manuscritos, así como la conveniencia de establecer una normativa para su descripción bibliográfica.

En esta institución, que todavía contaba con pocos años de existencia (su año fundacional data de 1712), se había ido creando un incipiente foco de profesionalidad bibliotecaria, promovido por personalidades tan destacadas como su director, Juan de Santander, y su bibliotecario de manuscritos, Juan de Iriarte, que en 1729 entra como escribiente en la institución, ascendiendo en 1732 a categoría de bibliotecario, cargo que conservaría hasta su muerte.

Es a este personaje a quien se deben, con toda probabilidad, las primeras normas de catalogación de manuscritos que datan, según la copia manuscrita conservada en la $\mathrm{BN}$, de 12 de agosto de $1762^{10}$. Estructuradas en 15 artículos, se insiste más en la descripción interna - título (art. 3; art. 5 a 7 sobre los procedimientos para averiguar el título de la obra); número de libros, partes y capítulos del manuscrito (art. 4); autor y copista (art. 8). Sin embargo, no se pasan por alto los datos externos, tales como los relativos a estado, forma, encuadernación, materia, letra, iluminaciones, foliación y fecha (todos estos datos incluidos en el art. 15); tipo de copia (art. 11) y notas sobre su propiedad (art. 9) y sus ediciones (art. 10).

Consecuencia de toda esta actividad, y debido a que la Biblioteca de El Escorial contaba ya con una muy buena colección de manuscritos en lengua árabe procedentes de diferentes colecciones, fue la magna publicación de esa época, obra del maronita libanés Miguel Casiri, de los manuscritos de la Biblioteca de El Escorial. Se trata de una soberbia obra en 2 volúmenes que lleva por título Bibliotheca Arabico-Hispana Escurialensis. Se publicó con una diferencia de 10 años, 1760 y 1770, casi dos siglos después de aquel primer inventario del secretario real Antonio Gracián (1572), donde se incluyen algunos códices árabes. Se trata de una obra que, aunque muy revisada, ha sido el sustento de posteriores estudios. Siguió el método de su también compañero maronita Giuseppe Assemani ${ }^{11}$.

Lo primero que hay que comentar de la obra de Casiri es que fue el "primer» catálogo que se puede considerar como tal, en el sentido de construcción científica. Supuso, además, una obra monumental, pues se basó para ello en la mejor colección de España. Afrontó con ello un reto importante y puso a disposición de los investigadores una referencia utilísima y asequible para conocer

\footnotetext{
${ }^{10}$ Han sido editadas con el título Instrucción para formar el índice de los manuscritos de la Real Biblioteca. Se encuentra en Vázquez de Parga, L., "Algunas noticias sobre la organización y primera catalogación de la Sección de manuscritos de la Biblioteca Nacional", Homenaje a Federico Navarro, [Madrid], 1973.

${ }^{11}$ Assemani, G., Bibliotheca Orientalis Clementino-Vaticana, Roma, 1719-1728, 3 vols.
} 
tal tesoro. Reflejo de su importancia es que, por ejemplo, se tradujo al árabe en Marruecos en 1811. Por todo ello, las críticas recibidas y las posteriores revisiones de que ha sido objeto no menguan en absoluto el valor de esta obra excepcional.

Del catálogo de Miguel Casiri, redactado en latín, conocemos los nombres de los autores a través de transliteración en el texto principal, seguido de la fecha de nacimiento. Sin embargo, siempre remite a una nota a pie de página (en este caso, pie de columna), donde el autor se consigna también en árabe. A continuación se cita el título de la obra "traducido al latín", con la correspondiente nota en árabe.

También se consignan en árabe y siempre a pie de página, los incipits (initium) y los explicit (finis), de manera tremendamente escueta (tan sólo dos o tres palabras). Consigna la fecha cuando la conoce y la mención se termina con comentarios y reproducciones ocasionales de textos en árabe. Se completa la obra con índices de autores y nombres geográficos al final del segundo volumen.

Los avances del siglo XVIII no tuvieron la deseable continuidad en la primera mitad del XIX, época tan tremendamente agitada de la historia de España y tan poco propicia al estudio y la erudición. De manera general, la difusión de los fondos manuscritos de las colecciones españolas a lo largo del siglo XIX fue sobre todo obra de eruditos extranjeros, llevada a cabo a través de las "misiones" o "viajes" científicos, que tan de moda estuvieron en la segunda mitad del pasado siglo.

Tenemos que esperar a la segunda mitad del siglo para empezar a encontrar noticias de catálogos. Han transcurrido ya más de 100 años desde la aparición de la obra de Casiri. En el último tercio del siglo XIX se publica el catálogo de Lafuente Alcántara (1862), que contiene una relación de 233 manuscritos árabes comprados en Tetuán e incorporados a continuación a la Biblioteca Nacional. El primer dato es el título, mencionado en árabe y traducido al español; sigue la mención del autor, en transliteración, para pasar a estudiar la descripción externa, el tamaño, escritura, fecha de copia, número de folios, división de la obra y comentarios sobre el autor con referencias bibliográficas. Incluye índices de nombres y títulos.

Codera (1884) publica la noticia de los manuscritos conservados actualmente en el Instituto de Filología, CSIC, de Madrid.

En el mismo año, 1884, aparece la primera entrega del estudio realizado por Hartwig Derenbourg de los manuscritos de El Escorial. Supone ya el segundo catálogo de esta colección y revisa a Casiri. Este trabajo se enmarca en la corriente de eruditos e investigadores arabistas extranjeros que viajaron a España en busca de manuscritos. Varios discípulos de Derenbourg, E. Lévi-Provençal y 
H. Renaud, continuaron en los años siguientes este segundo catálogo de los manuscritos de El Escorial, basándose siempre en los apuntes iniciales de Derenbourg.

Los españoles también viajaron al extranjero. Prueba de ello, además de la obra de Lafuente Alcántara, es la serie de artículos que Codera publicó en el Boletín de la Real Academia de la Historia, entre 1888 y 1897, y que dan noticia de 82 manuscritos en total, con entradas consecutivas en cada uno de ellos. Otra vez hay que esperar casi un siglo (1975), fecha en la que se publica el trabajo de Elías Terés sobre la Colección Gayangos, también de la Real Academia de la Historia.

El final del siglo XIX contempla la aparición del catálogo de los manuscritos de la Biblioteca Nacional de Madrid, calificado por Sánchez Mariana como de “totalmente asistemático». Tiene referencias a la obra de Lafuente Alcántara. Creo, sin embargo, que no podemos dejar de conceder importancia a esta obra, pues, entre otras cosas, es el primer y único catálogo de los manuscritos de la BNM.

Tras una interesante introducción, ordena los datos según el orden topográfico. La entrada principal de autor se cita en transliteración según el método propuesto por Leopoldo Eguílaz Yanguas ${ }^{12}$, algo incómoda de reconocer. Intenta resaltar también la parte del nombre más destacada. El título lo menciona en árabe aunque inmediatamente ofrece la traducción castellana, hecho que comprobamos se repite en numerosos ejemplos de posteriores catálogos. A continuación menciona la descripción externa, tamaño, número de folios, tipo de soporte, medidas de la caja de escritura, número de líneas de páginas, incipit, explicit, número de folios y letra.

Tras estos datos, se encuentra parte de la descripción interna, asunto de la obra, sus divisiones, somera biografía del autor, nombre del copista, fechas de la copia y noticia de su procedencia, así como ediciones de la obra. Inserta referencias bibliográficas, entre ellas Lafuente Alcántara y la obra de Pons Boigues ${ }^{13}$.

Vemos, pues, que sigue de cerca las instrucciones de Iriarte, aunque el resultado es algo deficitario.

Creo sin dudar que lo más valioso de este catálogo son los índices de autores, títulos en árabe y de materias.

A diferencia de El Escorial, los manuscritos de la Biblioteca Nacional no han sido objeto de una segunda catalogación completa; todo lo más, encontramos unas notas críticas realizadas por Derenbourg en 1904. Sin embargo, sí se han ido

\footnotetext{
${ }^{12}$ Eguílaz Yanguas, L., Estudios sobre el valor de las letras arábigas en el alfabeto castellano y reglas de lectura, Madrid, 1874.

${ }^{13}$ Pons Boigues, F., Ensayo bio-bibliográfico sobre los historiadores y geógrafos arábigoespañoles, Madrid, 1898.
} 
agregando nuevas noticias a la colección de Madrid, aparecidas, principalmente, en diversas revistas con un tono más moderno en su tratamiento ${ }^{14}$.

El trabajo de Almagro y Cárdenas (1899) sobre los manuscritos de la Universidad de Granada apareció publicado el último año del s. XIX, bajo las indicaciones del entonces Jefe de la Biblioteca. Incluye mención de autor, transliterado, y cuando está seguro lo destaca por la šuhra; sigue el título en árabe y su traducción (como Guillén Robles); para pasar a destacar el tamaño y tipo de soporte, incipit ("comienza»), explicit («concluye»), número de folios, escritura, tinta, color, notas marginales, tipo de encuadernación. Por último, ofrece un comentario extenso sobre lo que significa la obra, quién es el autor, y la fecha, si la hay. En general, es bueno.

Entrando ya en el siglo XX, podemos dividirlo en dos partes. En la primera parte del siglo, Pablo Gil y Gil describe (1904) la noticia de 40 manuscritos aljamiados que compró tras su descubrimiento en Almonacid de la Sierra, y que ahora están en el Instituto de Filología del CSIC.

A Miguel Asín Palacios se le debe, sin duda alguna, el mejor catálogo. En 1911 hace una descripción modélica de los manuscritos árabes del Sacro-Monte de Granada. Su metodología es precisa y clara. Comienza por el título en árabe, con indicación del folio del ms. donde se encuentra. Continúa con la mención del autor, también en árabe y desde el principio e indicando asimismo el folio del manuscrito donde se encuentra. A continuación la materia, una sola palabra basta, para pasar a consignar la época. El incipit ('comienza') y explicit ('acaba') se hace también en árabe. Bajo el epígrafe de «Descripción material» describe todo el apartado externo del manuscrito, para terminar con la identificación, comentario sobre el autor, lo que significa la obra y si existen otras copias y dónde. Destaca con mucho sobre el conjunto de las existentes.

De la misma colección del Instituto de Filología hay (1912) un detallado catálogo elaborado bajo la dirección de Ribera y Asín Palacios. Su valor principal reside en la presencia de índices de autores, títulos, topónimos árabes, nombres españoles, títulos aljamiados y en español y topónimos regionales. Existe reimpresión de 1928.

A partir de la segunda mitad del siglo XX encontramos noticias más modernas de diversas colecciones, donde la información suministrada se nos presenta, a veces, de una forma más científica.

En apenas cuatro páginas, Varela (1965) nos ofrece la noticia del descubrimiento de algunos manuscritos en el Ayuntamiento de Villarrubia de Santiago; Viguera (1974) aporta datos sobre autores y obras de la pequeña colección de la Fundación Lázaro Galdiano de Madrid; Terés (1975) diserta por temas sobre los

${ }^{14}$ Véase supra, nota 5. 
manuscritos de la Real Academia de la Historia; Epalza (1977) da datos breves, pero bastante completos, de la Biblioteca March de Palma de Mallorca, aunque la transcripción tiene que plegarse a las posibilidades tipográficas normales en castellano, y no resulta demasiada clara; Balañà (1979) ofrece un esquema que aplica en su trabajo y se aprecia.

De entre todos ellos destacan con mucho Braulio Justel, autor de varios catálogos, y Concepción Castillo (1984). Justel presenta en 1979/80 toda una declaración de intenciones para la catalogación del nuevo fondo de manuscritos de la Biblioteca Nacional y que se convertirá en leit-motiv de catalogaciones posteriores. Prefiere la transliteración a la presentación en árabe de los datos y procura añadir el lugar de donde ha tomado los datos, hecho bastante encomiable. Junto con Miguel Asín Palacios sus trabajos son los más acertados y siguen una línea bastante regular.

\section{NORMATIVA SOBRE CATALOGACIÓN}

A la hora de realizar un trabajo científico es necesario precisar, en primer lugar, qué normas seguir. Antes de continuar con el tema, me gustaría dar un repaso breve, pero general, a lo que supone el trabajo de catalogación, descripción bibliográfica e indización, englobado todo ello dentro del proceso de análisis documental ${ }^{15}$, técnica utilizada en la elaboración de catálogos de todo tipo de material bibliográfico.

El análisis documental es el conjunto de operaciones realizadas para representar el contenido de un documento de forma distinta de la original, con el fin de facilitar la consulta o la recuperación. Comprende la descripción bibliográfica, la catalogación, la indización y el resumen.

- Descripción bibliográfica. Es el conjunto de datos bibliográficos con que se identifica y registra un documento bibliográfico, de acuerdo con unas reglas. Tiene por objeto identificar al autor, el título de la contribución, etc. Se siguen para ello las normas de las ISBD, descripción bibliográfica normalizada internacional, que dividen en zonas los datos que interesa tomar. Las ISBD suponen un conjunto de normas aceptado internacionalmente para la presentación de los datos bibliográficos en los asientos.

\footnotetext{
${ }^{15}$ Los datos que vienen a continuación están tomados de Martínez Sousa, J., Diccionario de bibliología y ciencias afines, Salamanca, 1989.
} 
Completamente desarrolladas para la descripción de asientos bibliográficos de monografías, publicaciones periódicas, materiales gráficos y otros, y aunque existe una ISBD para manuscritos, es difícil su adaptación para el tratamiento de manuscritos en lengua árabe.

- Catalogación. Es la representación normalizada de los datos documentales para la localización física de los documentos ordenados en catálogos según criterios determinados. Es un proceso técnico que tiene por fin sistematizar el conjunto bibliográfico, de acuerdo con unas normas, para darle unidad y coherencia. Comprende dos pasos: la redacción del asiento bibliográfico primero y la formación del catálogo mediante la ordenación de aquellos, después.

- Indización. Aunque también se puede referir a la confección de índices, en este proceso de análisis documental se alude a la extracción de conceptos del texto de un documento para expresarlos con la ayuda de un lenguaje, como palabras clave, descriptores o índices de una clasificación.

- Resumen. Es la representación condensada del contenido de un documento, generalmente trabajos, artículos o contribuciones de publicaciones periódicas, mediante técnicas de indización y análisis. Tiene su utilidad en bases de datos de artículos.

El producto del análisis documental es un documento secundario que representa sintéticamente al original, contiẹne una información concentrada, puede ser consultado más fácilmente y ofrece las primeras noticias de existencia de los documentos primarios.

En el plano oficial y ya contemplando la normativa sobre manuscritos, al menos en España, en 1910 y en Madrid se publican las Instrucciones para la catalogación de manuscritos, estampas, dibujos originales, fotografías y piezas de música de las bibliotecas públicas, redactadas por la Junta Facultativa del Ramo. Las normas son breves, ajustadas y precisas, y contienen reglas especiales para cartas, piezas de teatro, tomos de poesía, etc. Supusieron la divulgación de una normativa a la que se debían atener los que de ahí en adelante redactasen catálogos de manuscritos.

En 1957 hubo un intento de publicación de normas, llevado a cabo por Pedro Longás, bibliotecario de manuscritos de la Biblioteca Nacional. Ese año presentó una "Ponencia sobre catalogación de manuscritos" en el Congreso Iberoamericano y Filipino de Archivos, Bibliotecas y Propiedad Intelectual. Longás insiste en la descripción externa del manuscrito y establece un orden: foliación, clase de manuscrito, letra del manuscrito y fecha, tamaño, materia escriptoria, notas del manuscrito, miniatura, procedencia, y su bibliografía. 
También en 1957 se publican las Instrucciones para la catalogación de manuscritos que recogen toda la experiencia anterior. Se trata de unas normas de tipo general y suponen el mayor esfuerzo por establecer una normativa coherente y moderna. El inventario de manuscritos de la Biblioteca Nacional de Madrid se ha basado en estas normas.

En España este conjunto de normas de catalogación contemplaron un avance considerable en 1985, cuando se publica la primera edición de las Reglas de catalogación, considerada la cuarta versión de las reglas oficiales por las que se rige la tarea de identificación de un libro entre todos los materiales bibliográficos y documentales de una biblioteca.

De 1995 data la primera edición, corregida y aumentada. Incorpora ya, en un solo volumen, la descripción (familia de las ISBD) de las distintas clases de materiales en aquellos puntos en los que la descripción difiere de las reglas generales. El capítulo 3 está dedicado a la "Descripción de manuscritos", correspondiendo el capítulo 14 a la "Elección de puntos de acceso" y el capítulo 15 a la "Forma de encabezamiento". Todo lo relativo al "Título uniforme" se desarrolla en el capítulo 16, las "Referencias" en el capítulo 17 y los apéndices contienen la ayuda indispensable para títulos uniformes, formas de autores clásicos griegos y latinos, información sobre órdenes y congregaciones religiosas y sus siglas, transliteraciones ${ }^{16}$ e información de términos de abreviaturas en diferentes idiomas.

La inusitada explosión de la información ha hecho que el uso de la informática para el tratamiento bibliográfico se haya desarrollado de forma considerable en los últimos años. Los primeros estudios e intentos fueron desarrollados por la americana Library of Congress y, cuatro décadas después de sus primeras observaciones, casi todos los programas automatizados incluyen alguna variante de lo que se conoce por formato Marc (Machine Readable Cataloguing, norma ISO 2709). Se trata de un formato normalizado, dividido en campos y subcampos, en los que se procede a dar entrada a los datos bibliográficos una vez que se ha procedido al análisis de catalogación y descomposición de la descripción bibliográfica. Finalizadas las operaciones pertinentes, todos los sistemas automatizados ofrecen la posibilidad de escoger una visualización de la ficha final de acuerdo a la descripción ISBD, de acuerdo a la disposición del mismo formato Marc, con sus campos y subcampos o de acuerdo con una forma más accesible y amigable al usuario final.

Desde esta perspectiva y teniendo en cuenta todo lo anterior, diversas voces expertas indican que lo mejor es que el catalogador tendrá que irse creando su

\footnotetext{
${ }^{16}$ Respecto a la árabe, menciona la de la Escuela de Estudios Árabes de Granada.
} 
propia normativa y, también, deberá tener unas nociones previas del fondo para, conociendo la normativa existente al respecto, aplicarla y/o adaptarla a la naturaleza y característica del fondo a catalogar. Lo que es muy claro es que la catalogación de manuscritos no puede efectuarse igual que la de un impreso, tal y como hacemos ahora, pero el grado de la técnica y de los conocimientos deben ser los mismos.

Llegados ya a este punto, me voy a referir a continuación a aspectos muy concretos que tienen relación con lo dicho anteriormente y que pueden ser de aplicación en la catalogación de manuscritos en lengua árabe, intentando compaginar todas las experiencias, provenientes de los diferentes campos de actuación. Cada uno puede aplicar un estilo propio de aplicación, pero con un fundamento que lo respalde.

En primer lugar, habría que decidir cómo se hace la descripción, bien en la escritura original (así lo aconsejaba Iriarte), bien en transliteración y, si es elegida esta segunda alterntiva, cuál adoptar. También se pueden combinar entradas, algunas en escritura original, otras en transliteración.

Después tenemos que pensar sobre:

- Datos topográficos y signatura. Ciudad, nombre de la biblioteca que aloja la colección y número de orden del manuscrito que le identifique entre la colección.

Si se está trabajando sobre una colección única que se conserva alojada en un mismo sitio, los datos de ciudad y biblioteca no son necesarios repetirlos continuamente. Sí es necesario, por el contrario, que se encuentre destacado el número de orden, signatura u otra numeración que identifique claramente al manuscrito.

En un catálogo impreso puede (casi hay que leerse como debe) colocarse al principio de toda la información de manera que sea éste el orden de entrada de los datos, en vez de hacerlo por materias ${ }^{17}$.

Muy importante es el olim, término latino que alude a la antigua signatura del volumen manuscrito.

- Entrada principal por el autor (al-mu'allif) ${ }^{18}$. La forma para consignar una entrada principal de autor en un catálogo, ya sea en árabe o en transliteración, debe hacerse de manera normalizada. Éste es un principio básico de todo proceso de catalogación ${ }^{19}$.

\footnotetext{
${ }^{17}$ Formato Marc campo 852.

${ }^{18}$ Formato Marc campo 100 \$a, o simplemente “Título:».

${ }^{19}$ Según el punto 15.1 de las Reglas de catalogación.
} 
Debe evitarse dar datos onomásticos exhaustivos, ya que no se trata de un fichero biográfico, aunque se puede estudiar añadir elementos informativos que pueden completar la información del nombre, por ejemplo, fechas de nacimiento y muerte, si la indicación de la entrada autorizada no es suficiente y es susceptible de confundirse con otro autor de nombre similar.

Sí se puede indicar en nota, si se considera necesario, de dónde se ha tomado esa entrada autorizada, ya sea de una fuente primaria (Brockelmann, Sezgin, Kaḥhāla, Ziriklī), como de una secundaria (algún tipo de catálogo o lista de autoridad).

Esta entrada, que debe ser única en el catálogo, debe referirse tanto a obras impresas como a manuscritas. Una biblioteca o colección puede disponer tanto de textos manuscritos de Ibn 'Arabī como de traducciones al español u otros idiomas de obras de este autor. La entrada en el catálogo, ya sea automatizado, impreso - en forma de volumen o artículo- o en fichas, debe ser única.

Respecto a las autoridades de nombres árabes, las reglas de catalogación no son claras en este punto, por lo que hay que recurrir al punto de vista de los propios interesados o científicos. Aunque hay poca bibliografía se puede consultar también otro tipo de obras, catálogos de bibliotecas impresos por ejemplo, donde se puede ver la actuación sobre este punto en otros centros con problemas similares.

En principio, se puede establecer que la entrada al catálogo para autores árabes puede efectuarse por la šuhra, es decir, la parte del nombre por el que el autor es más conocido, completándose después y tras coma, por el resto del nombre, comenzando por el ism y el nasab. Esta actuación responde plenamente al principio de las Reglas de catalogación ${ }^{20}$, por lo que no es tan descabellada su aplicación. Hemos visto además que autores como Guillén Robles o Almagro Cárdenas efectúan las entradas al catálogo por la parte del nombre más conocida. De la misma manera, Guillén Robles afirma en la introducción a su catálogo:

Los nombres de autores se citan empezando siempre por el más conocido, Almakari, Algazzali, Albeitar ó Assenusi; á éste sigue el «lakab", ó sobrenombre honorífico, Lissaneddin, Nureddin, Xemseddin; á este sigue la "cunia", ó apellido de ascendencia, Abu Chafar, Abu Abdallah, tras de lo cual va el nombre propio, Mohamed, Yahya, Soleimán, etc., y en pos de él los de la cunia de descendencia; todos ellos, algunos, ó solo uno, según haya podido averiguarse ${ }^{21}$.

\footnotetext{
${ }^{20}$ Según el punto 15.1.1 A Regla general.

${ }^{21}$ Cf. Guillén Robles, op. cit., VII.
} 
Todo ello nos indica una cierta tendencia a la normalización de los nombres. Creo que es bastante encomiable y no he encontrado nada parecido que haga referencia a problema tan complicado.

En subsiguientes catálogos a los de Guillén Robles comprobamos, por ejemplo, que aparece el nombre completo, empezando por la kunya o por el ism correspondiente, y al llegar a la šuhra se destaca tipográficamente, bien con mayúscula, o en cursiva o en negrita, lo que puede entorpecer la lectura o presentación visual del texto.

Las entradas autorizadas en el catálogo deben contemplarse en correspondientes ficheros de autoridades, que ya no son tan raros de localizar. En él constan las diferentes variantes que existen o pueden utilizarse de un encabezamiento de autor con la versión aceptada, remitiendo las versiones no aceptadas a la aceptada. Las formas no autorizadas en el fichero de autoridades deberían incluir todas las variantes del nombre del autor, una de las cuales puede empezar por la kunya. Una obra muy interesante en este sentido es el trabajo en 4 volúmenes de Fakrī al-Ŷazzār ${ }^{22}$.

- Entrada principal bajo un título uniforme $e^{23}$. Aunque es tarea algo delicada, si se trata de colecciones de manuscritos se puede adoptar un título colectivo ficticio como Miscelánea. El término árabe puede ser Maŷmü'a. También puede asignarse una denominación topográfica expresada por el nombre del depósito, seguido de la palabra "manuscritọ" y de la signatura topográfica que corresponda dentro de dicho depósito ${ }^{24}$.

De la misma manera se podría pensar en adoptar un título uniforme para el Corán, que o bien puede ser el mismo término $\operatorname{Corán}^{25} \mathrm{o}$, por el contrario, puede ser la palabra transliterada - Qur'ān-, descartando otros como Alcorán, o Al-Qur'ān, formas todas ellas aparecidas en los catálogos estudiados.

Las obras cuyo autor se desconoce pueden llevar un título uniforme que responda al término "Anónimo", aunque no es necesario en absoluto.

- Título uniforme [bajo autor conocido] (al-'unwān al-muwaḥhad). En catalogación, es el título adoptado para catalogar una obra que se conoce por varios títulos, generalmente establecido siguiendo unas normas. Tiene como

${ }^{22} \mathrm{Al}$-Ŷazzār, F., Madāil al-mu'allifin wa-l-a 'lām al-'arab: hattà 'ām $1215 \mathrm{~h}=1800 \mathrm{~m}$, Al-Riyāḍ, 1991-1994.

${ }^{23}$ Formato Marc campo $130 \$ a$.

${ }^{24}$ Según 16.4.1 f) de las R.C.

${ }^{25}$ Según 16.7.2 de las R.C., el término a adoptar es Corán. 
principio básico recoger en el catálogo todas las variantes bibliográficas de una misma obra. Debe hacerse referencia en nota a la fuente de donde se ha tomado la normalización.

Este principio, muy útil en otras descripciones bibliográficas, no está nada desarrollado en descripciones de manuscritos en lengua árabe, aunque no debe descartarse. Por ejemplo, un catalogador se podría plantear el problema que ocasiona el término kitāb al comienzo de la mención de un título. De todos es sabido que tal palabra muchas veces no es indicativa ni significativa del título. En muchos catálogos, observamos menciones tales como K. Ŷămi ‘ al-adwiya o K. ișlāh al-mantiq, lo que manifiesta una cierta incomodidad con esta palabra.

De la misma manera se podría pensar en la posibilidad de añadir la mención de lengua en la que se encuentra el texto, si no es en árabe, o el término "Aljamiado" para todos estos manuscritos.

Un hecho que se observa es que, de la misma manera que sí hay esfuerzos por normalizar la entrada por autor, no conozco ningún intento de normalización de títulos uniformes de obras en lengua árabe.

- Título propiamente dicho (al- 'unwān $)^{26}$. Es principio básico de la descripción bibliográfica que el título "debe transcribirse tal y como se encuentre en la fuente", es decir, en nuestro caso, tal y como se encuentre en el mismo manuscrito.

Este título puede encontrarse bien en la primera página del manuscrito, bien en el incipit - las primeras líneas donde se dan noticia del autor y título-, bien al final del texto, etc. Sin embargo, y en la tipología de los manuscritos que estamos tratando, es muy frecuente que se pueda encontrar en las primeras páginas del texto, en medio o al final del texto, tras la expresión wa-samaytu-hu..., y es el texto a continuación el que se debe transcribir en la descripción bibliográfica, «independientemente» de cuál sea el título que ofrecen las obras de referencia.

Si el título se toma del tejuelo puede servirnos, aunque hay que ponerlo entre corchetes, de la misma manera que si se encuentran variantes del título a lo largo del manuscrito, deben consignarse y anotar su procedencia en nota. Si no hay ningún título, debemos adoptar uno y hacerlo constar entre corchetes.

${ }^{26}$ Formato Marc $245 \$ a$. 
- Mención de responsabilidad. Se confunde a menudo este apartado con el referido a la entrada principal de autor. En este punto estamos hablando de descripción bibliográfica y «debe registrarse la mención de responsabilidad que aparezca en el manuscrito ${ }^{27}$, a diferencia de la forma en que aparecerá bajo el catálogo, que ya hemos visto que corresponde a una forma autorizada y normalizada del autor. De esta manera se pueden ver las diferentes formas y variantes de nombres que aparecen en los distintos manuscritos, especialmente ahora con las nuevas tecnologías, que no tendrán dificultad en recoger las variantes onomásticas.

Puede consignarse en nota de dónde se toma la mención.

Así como en los libros y ediciones modernos tienen mucha importancia los editores de textos, traductores y demás formas de colaboración científica en un texto clásico, en nuestro caso cobra una importancia muy principal la figura del copista (al-nāsij), cuya mención suele encontrarse al final del manuscrito, en el explicit.

En cualquier caso, debe hacerse constar tras el autor, mejor que en nota.

- Lugar (al makān) ${ }^{28}$. Perteneciente a la descripción externa hay que tener cuidado en no equivocar el lugar mencionado en la fuente con el lugar de la copia y que tiene una relación muy clara con el siguiente dato, el de la fecha. Se debe consignar tal y como se encuentre en la fuente, si se quiere en transliteración. [Fās, Bagdād, Išbīliya, etc.]

- Fecha $(a l-t a ' r \ddot{j})^{29}$. Pertenece al apartadó de lo que se llamaría descripción externa. Es mención sumamente interesante, ya que en los manuscritos de los que estamos hablando, la mención de la fecha estará consignada en término de cómputos no cristianos y como tal hay que consignarlo en la descripción, pudiéndose en nota hacer referencia a la equivalencia en términos cristianos. Es importante destacar si se refiere primero a la terminación del manuscrito original y después al de la copia. Si no hay mención de fecha en el manuscrito, es deseable que aparezca una con la máxima aproximación, si acaso con indicación del siglo.

- Extensión y designación específica de material ('adad al-awrāq). Marca la tradición que en los manuscritos se hable de foliación y no de paginación. Si no hay indicación de tal debe hacerse, siempre con lápiz y en la esquina

\footnotetext{
${ }^{27}$ Según 3.1.7 de las R.C.

${ }^{28}$ Formato Marc $260 \$$ a.

${ }^{29}$ Formato Marc 260 \$c.
} 
superior izquierda, respetando si hay otras paginaciones varias. Se debe empezar a foliar desde la misma primera hoja del texto y las hojas en blanco también son susceptibles de foliación, indicando siempre dónde empieza y acaba un texto o los textos posteriores.

Aquí tienen cabida el número de columnas de la página del texto y el número de líneas por página, datos éstos que generalmente se suelen citar en $\operatorname{nota}^{30}$.

- Otras características físicas. Se refiere este apartado a la mención del soporte sobre el que está copiado el manuscrito, si es distinto de papel. También si se trata de códices especialmente valiosos con ilustraciones.

— Tamaño del manuscrito. Se indica en cm, como en el material impreso.

- Tras los datos anteriores se llega al apartado de notas, que debe iniciarse con el incipit. Supone la transcripción de tantas palabras del comienzo de la parte principal del texto como sean necesarias para la identificación de la pieza. En los manuscritos árabes que suelen empezar con la basmala, se puede utilizar la expresión wa-ba'da l-basmala para consignar el texto que sigue inmediatamente después.

- Se sigue con el explicit: últimas palabras del texto.

- Materia o indicación del contenido del texto. Debe hacerse de forma abreviada, concisa y clara.

- Notas después de la descripción bibliográfica. Una buena catalogación debe completarse con notas, referido a todo aquello que haya podido quedar incompleto en la descripción anterior, por ejemplo, clase de manuscrito, tipo de escritura, procedencias anteriores, procesos de transmisión, relación de inventarios donde aparezca recogido el manuscrito, vía de entrada en la biblioteca y fecha. También se puede hacer constar el número de cuadernos, reclamos, signaturas, encuadernación y número de microfilm si está microfilmado, lo que facilitará sobremanera la petición de reproducciones, y bibliografía, con ediciones de los que haya sido objeto, y catálogos donde haya sido descrito y estudios de los que haya sido objeto.

\footnotetext{
${ }^{30}$ Por ejemplo: 310 ff ( 2 columnas; $15-17$ líneas), siempre según 3.5.3 de las R.C.
} 
Si se opta por catálogos impresos, hay que conceder especial importancia a los índices, para facilitar el acceso a la información por todos los puntos de acceso posibles, autor, título, copistas, fechas, lugares, etc.

Dando la bienvenida ya al siglo XXI e inmersos completamente en la era tecnológica, no podemos sino pensar en organizar la información de nuestro legado cultural de manera precisa con la adopción de unos criterios bien definidos, apostando por la tradición bibliográfica y catalográfica, e intentando compaginarla con las peculiaridades de los materiales a tratar.

Una base de datos bien diseñada con entradas muy claras y bien definidas será el instrumento más útil para el conocimiento de un legado cultural que no tenemos nunca que desatender si no queremos perderlo para siempre.

\section{RESUMEN}

El presente estudio se basa en las colecciones de los manuscritos árabes que se conservan actualmente en España. Para ello se hace un análisis de las colecciones existentes. A continuación se lleva a cabo un estudio de los catálogos e inventarios aparecidos, desde los primeros en el siglo XVI hasta la actualidad, deteniéndose en los más destacados.

La segunda parte del estudio se centra en lá técnica del análisis documental, que engloba los trabajos de catalogación y descripción bibliográfica. Se repasa la normativa vigente, para terminar con reflexiones personales de los datos que deben aparecer en los catálogos y cómo deben aparecer.

\section{ABSTRACT}

The present study is based on Arabic manuscripts presently conserved in Spain, for which an analysis of existing collections is made. Then a study of the catalogues and inventories is carried out which have appeared since the beginning of the 16th century to the present, treating the most important of them.

The second part of the study centers on the method of documentary analysis covering both cataloguing and bibliographical description. Prevailing guidelines are reviewed, concluding with personal reflexions as to what data should appear in the catalogues and how they should do so. 